\title{
Energy and multiplicity dependence of strange and non-strange particle production in proton-proton collisions at the LHC with ALICE
}

\author{
Fiorella Maria Celeste Fionda ${ }^{1}$, on behalf of the ALICE Collaboration ${ }^{\star}$ \\ ${ }^{1}$ University of Bergen (Norway)
}

\begin{abstract}
The study of energy and multiplicity dependence of hadron production in proton-proton collisions provides a powerful tool to understand similarities and differences between small and large colliding systems. In this work we present mid-rapidity measurements of the $p_{\mathrm{T}}$ spectra and yields of identified hadrons, namely pions, kaons, protons, $\mathrm{K}_{\mathrm{S}}^{0}, \Lambda, \Xi$ and $\Omega$ in pp collisions at $\sqrt{s}=7$ and $13 \mathrm{TeV}$. The comparison of results at $\sqrt{s}=13 \mathrm{TeV}$ to earlier results at $7 \mathrm{TeV}$ provides insights about the energy dependence of the strangeness enhancement. Comparisons between data and expectations from commonly-used Monte Carlo event generators will be presented.
\end{abstract}

The primary goal of the ALICE experiment is the study of the strongly-interacting Quark-Gluon Plasma (QGP), which is expected to be produced in ultra-relativistic heavy-ion collisions. In order to interpret correctly heavy-ion results proton-proton (pp) and proton-nucleus ( $\mathrm{p}-\mathrm{A}$ ) collisions ("small" systems) are used as a reference and control experiments. However, in recent measurements from the highest LHC energies, it was observed that small systems showed striking commonalities with heavy-ion collisions when multiplicity dependent studies are performed. Several effects, like near-side long-range correlations and mass-dependent hardening of $p_{\mathrm{T}}$ distributions, which in nuclear collisions are typically attributed to collectivity, have been observed in high-multiplicity pp and p-A collisions at the LHC (see for example [1,2]). These commonalities raise questions about the possibility to employ a description based on the assumption of thermal and chemical equilibrium, commonly used in the heavy-ion context, also in small systems. The study of identified hadron production, both integrated yields and differential $p_{\mathrm{T}}$-spectra, as a function of the charged particle multiplicity represents a powerful tool to shed light on these open points. In these proceedings a selection of multiplicity dependent results obtained by ALICE at mid-rapidity will be discussed.

A detailed description of the ALICE apparatus and of its performance can be found in [3, 4]. The main subdetectors used to measure identified hadrons at mid-rapidity are: (i) the Inner Tracking System (ITS) which is composed of six cylindrical layers of silicon detectors employing three different technologies; (ii) the Time Projection Chamber (TPC) which provides track reconstruction with up to 159 three-dimensional space points per track, as well as particle identification (PID) via the measurement of the specific energy deposit $\mathrm{d} E / \mathrm{d} x$ in the gas; (iii) the Time-Of-Flight detector (TOF), a cylindrical detector equipped with Multi-gap Resistive Plate Chambers (MRPCs) dedicated to PID. The V0 detector consists of two arrays of 32 scintillators, each placed on both sides of the interaction

^e-mail: fiorella.fionda@cern.ch 
region and covering the pseudo-rapidity intervals of $-3.7<\eta<-1.7$ and $2.8<\eta<5.1$. It is used for triggering as well as to define multiplicity event classes based on the total charge deposited in the scintillators. For each event class the average pseudorapidity density of primary charged particles $\left\langle\mathrm{d} N_{\mathrm{ch}} / \mathrm{d} \eta\right\rangle_{|\eta|<0.5}$ is measured at mid-rapidity $(|\eta|<0.5)$.

The transverse momentum distributions for several particle species measured at mid-rapidity $(|y|<0.5)$ in pp collisions at $\sqrt{s}=7 \mathrm{TeV}$ are shown in Fig. 1, for different event multiplicity selections, with class I denoting the highest multiplicity and class $\mathrm{X}$ the lowest. Transverse momentum spectra are observed to become harder as charged particle multiplicity increases, with changes of the spectra happening at low- $p_{\mathrm{T}}$ and being more pronounced for heavier particles. In heavy-ion collisions this behaviour is interpreted in terms of collective expansion (radial flow) which may require the system to be in local thermodynamical equilibrium. It is possible to further investigate

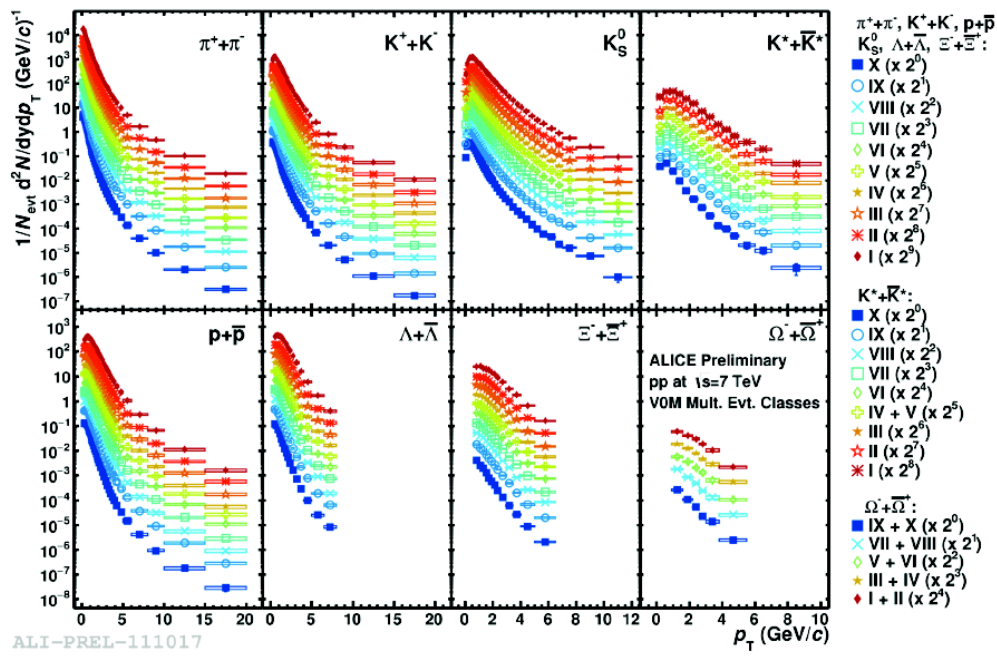

Figure 1. $p_{\mathrm{T}}$-differential spectra for several particle species plotted for different event multiplicity classes (indicated by different symbols and colours). The different spectra from the various event multiplicity selection are scaled by a of factor $2^{n}$ in order to improve visibility.

the multiplicity dependence of $p_{\mathrm{T}}$-spectra and the hypothesis of the collective expansion by studying the baryon-to-meson ratio as a function of the transverse momentum. The $\mathrm{p} / \pi$ ratio as a function of $p_{\mathrm{T}}$ is shown in Fig. 2 for $\mathrm{pp}(\sqrt{s}=7 \mathrm{TeV}), \mathrm{p}-\mathrm{Pb}\left(\sqrt{s_{\mathrm{NN}}}=5.02 \mathrm{TeV}\right)$ and $\mathrm{Pb}-\mathrm{Pb}\left(\sqrt{s_{\mathrm{NN}}}=2.76\right.$ $\mathrm{TeV}$ ) collisions at both low and high multiplicity. The ratio at high multiplicity in pp collisions exhibits a characteristic depletion at low- $p_{\mathrm{T}}(\sim 0.7 \mathrm{GeV} / c)$, an enhancement at intermediate- $p_{\mathrm{T}}(\sim 3$ $\mathrm{GeV} / c$ ) and no modification at high- $p_{\mathrm{T}}$ if compared to the corresponding results at low multiplicity. This behaviour is qualitatively similar to the one observed in $\mathrm{p}-\mathrm{Pb}$ and $\mathrm{Pb}-\mathrm{Pb}$ collisions. From a quantitative point of view the observed changes in these particle ratios are very different in the various colliding systems. However it is important to take into account that also the corresponding average charged particle multiplicity densities at mid-rapidity differ significantly. Studying carefully the measured ratios as a function of $\left\langle\mathrm{d} N_{\mathrm{ch}} / \mathrm{d} \eta\right\rangle_{|\eta|<0.5}$ in specific $p_{\mathrm{T}}$ intervals, a remarkable smooth trend across all colliding systems is observed. This is shown in Fig. 3.

Concerning the hadrochemistry one of the most intriguing results recently reported by the ALICE 


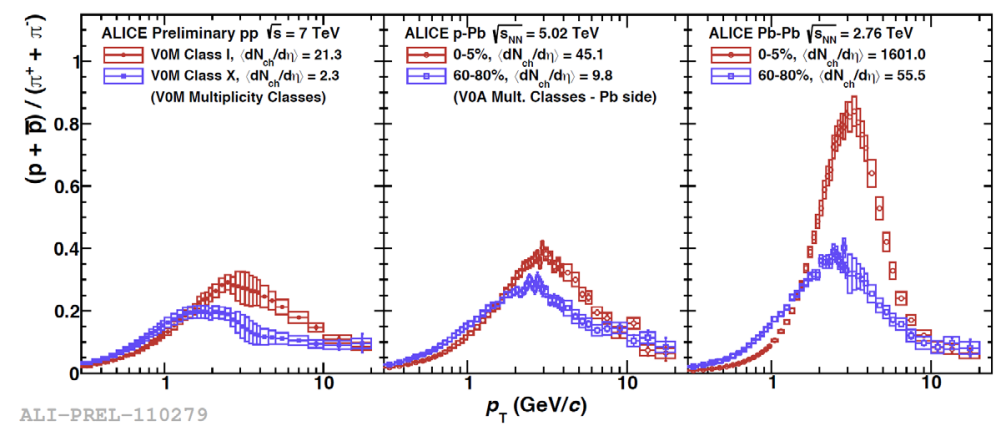

Figure 2. $\mathrm{p} / \pi$ ratio as a function of $p_{\mathrm{T}}$ in $\mathrm{pp}$ (left), $\mathrm{p}-\mathrm{Pb}$ (middle), $\mathrm{Pb}-\mathrm{Pb}$ (right) collisions for high and low multiplicity intervals.

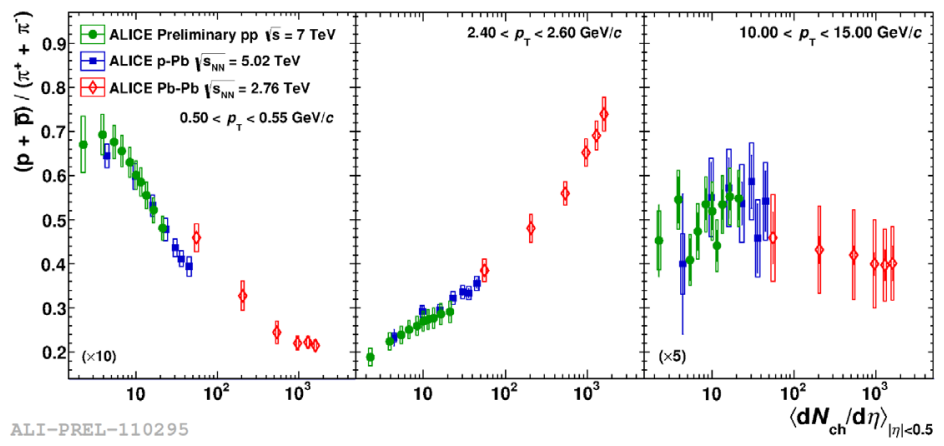

Figure 3. $\mathrm{p} / \pi$ ratio in low, mid and high- $p_{\mathrm{T}}$ intervals in $\mathrm{pp}, \mathrm{p}-\mathrm{Pb}, \mathrm{Pb}-\mathrm{Pb}$ collisions as a function of $\left\langle\mathrm{d} N_{\mathrm{ch}} / \mathrm{d} \eta\right\rangle_{|\eta|<0.5}$.

collaboration is the enhancement observed in the production of (multi-)strange hadrons relative to pions as a function of multiplicity in pp collisions at $\sqrt{s}=7 \mathrm{TeV}$ [5]. These are surprising observations, because strangeness enhancement was considered historically one of the signatures for the deconfinement in heavy-ion collisions [6], and also because none of the commonly-used pp Monte Carlo models reproduces quantitatively the existing data. In particular, QCD-inspired models such as PYTHIA [7, 8] predict a flat dependence of strange-to-pion ratios as a function of multiplicity. Models that include effects from densely packed strings as implemented in DIPSY [9], core-corona mechanism as in EPOS [10] or statistical-thermal models implementing strangeness canonical suppression [11] can reproduce qualitatively the observed trends.

The energy dependence of the strange hadron yields as a function of $\left\langle\mathrm{d} N_{\mathrm{ch}} / \mathrm{d} \eta\right\rangle_{|\eta|<0.5}$ is reported in Fig. 4, where multiplicity dependent results in pp collisions at $\sqrt{s}=7$ and $13 \mathrm{TeV}$ are compared. The yields of strange hadrons increase linearly with the charged particle multiplicity, and the production rate is the same at the two energies for a given multiplicity. It should also be noted that the yields of particles with larger strange quark content increase faster as a function of multiplicity as already reported in [5]. Data are compared to several Monte Carlo models. The comparison shows clearly that both PYTHIA 6 and PYTHIA 8 are not able to describe the multiplicity dependence of strange particle yields, and the disagreement increases with the strangeness content of the considered hadron. EPOS predicts the strangeness enhancement but it overestimates the increase of multi-strange hadrons 

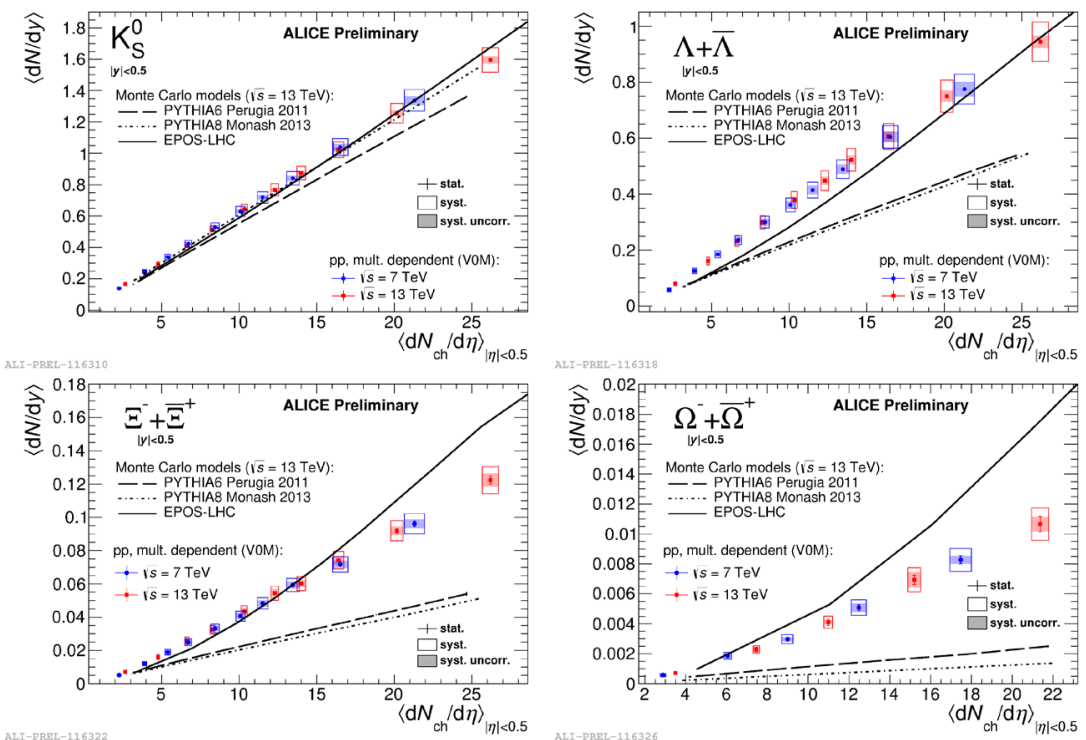

Figure 4. Integrated yields of $\mathrm{K}_{\mathrm{S}}^{0}, \Lambda, \Xi$ and $\Omega$ as a function of $\left\langle\mathrm{d} N_{\mathrm{ch}} / \mathrm{d} \eta\right\rangle_{|\eta|<0.5}$ in pp collisions at $\sqrt{s}=7$ and 13 $\mathrm{TeV}$. The results are compared to predictions from several Monte Carlo models.

at high multiplicity.

In summary, ALICE provides a comprehensive set of results on the production of identified hadrons as a function of charged particle multiplicity in pp collisions. These results show striking commonalities with heavy-ion collisions, and none of the tested Monte Carlo models succeeds to quantitatively reproduce the data. These results provide an important input to the theory community for further tuning or implementation of new model ingredients, in order to improve significantly our knowledge about the microscopic mechanisms that are responsible of these intriguing features observed in small systems.

\section{References}

[1] CMS Collaboration, V. Khachatryan et al., JHEP 09091 (2010).

[2] ALICE Collaboration, B. Abelev et al., Phys. Lett. B 728 25-38 (2014).

[3] ALICE Collaboration, K. Aamodt et al., JINST 3 S08002 (2008).

[4] ALICE Collaboration, B. Abelev et al., Int. J. Mod. Phys. A 291430044 (2014).

[5] ALICE Collaboration, J. Adam et al., Nature Phys. 13 535-539 (2017).

[6] J. Rafelski and B. Müller, Phys. Rev. Lett. 481066 (1982).

[7] T. Sjostrand, S. Mrenna, and P. Z. Skands, JHEP 05490 (2006).

[8] P. Skands, S. Carrazza, and J. Rojo, Eur. Phys. J. C 743024 (2014).

[9] C. Bierlich, G. Gustafson, L. Lnnblad, and A. Tarasov, JHEP 03148 (2015).

[10] T. Pierog, I. Karpenko, J. Katzy, E. Yatsenko, and K. Werner, Phys. Rev. C 92034906 (2013).

[11] V. Vislavicius and A. Kalweit, arXiv:1610.03001 [nucl-ex]. 